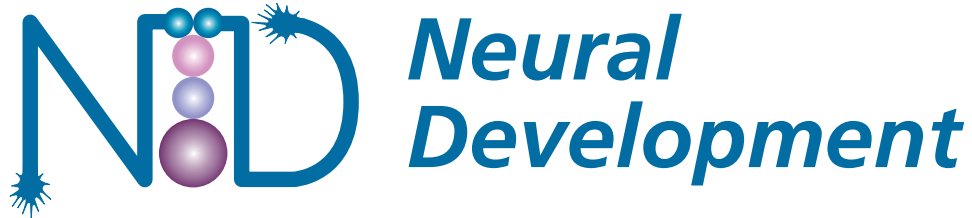

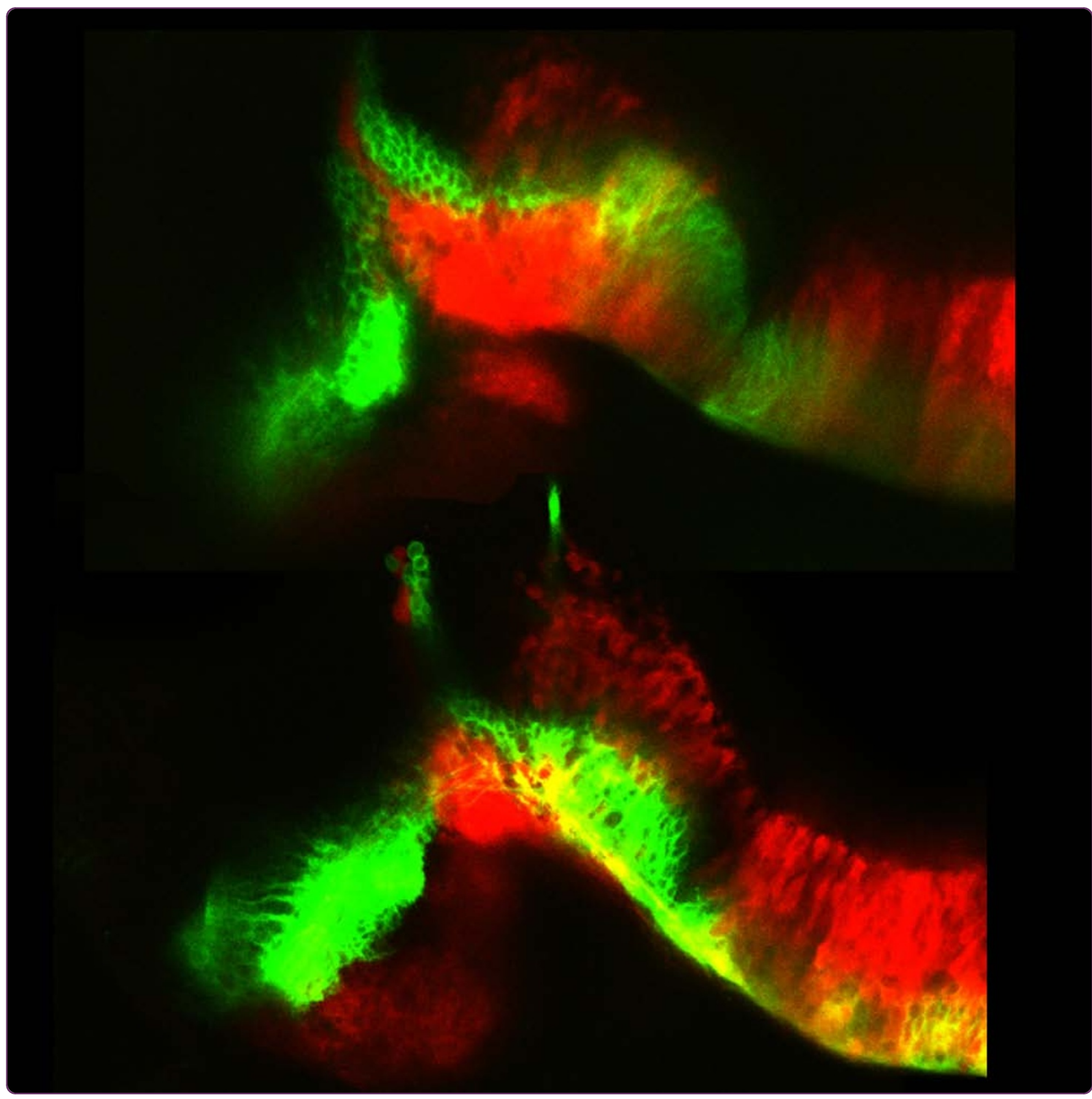

Wnt3 and Wnt3a are required for induction of the mid-diencephalic organizer in the caudal forebrain

Mattes et al. 


\title{
Wnt3 and Wnt3a are required for induction of the mid-diencephalic organizer in the caudal forebrain
}

\author{
Benjamin Mattes ${ }^{1}$, Sabrina Weber ${ }^{1}$, João Peres ${ }^{2}$, Qing Chen ${ }^{1}$, Gary Davidson ${ }^{1}$, Corinne Houart ${ }^{2}$ and \\ Steffen Scholpp ${ }^{1 *}$
}

\begin{abstract}
Background: A fundamental requirement for development of diverse brain regions is the function of local organizers at morphological boundaries. These organizers are restricted groups of cells that secrete signaling molecules, which in turn regulate the fate of the adjacent neural tissue. The thalamus is located in the caudal diencephalon and is the central relay station between the sense organs and higher brain areas. The middiencephalic organizer $(M D O)$ orchestrates the development of the thalamus by releasing secreted signaling molecules such as Shh.
\end{abstract}

Results: Here we show that canonical Wnt signaling in the caudal forebrain is required for the formation of the Shh-secreting MD organizer in zebrafish. Wnt signaling induces the MDO in a narrow time window of 4 hours between 10 and 14 hours post fertilization. Loss of Wnt3 and Wnt3a prevents induction of the MDO, a phenotype also observed upon blockage of canonical Wnt signaling per se. Pharmaceutical activation of the canonical Wnt pathways in Wnt3/Wnt3a compound morphant embryos is able to restore the lack of the MDO. After blockage of Wnt signaling or knock-down of Wnt3/Wnt3a we find an increase of apoptotic cells specifically within the organizer primordium. Consistently, blockage of apoptosis restores the thalamus organizer MDO in Wnt deficient embryos.

Conclusion: We have identified canonical Wnt signaling as a novel pathway, that is required for proper formation of the $M D O$ and consequently for the development of the major relay station of the brain - the thalamus. We propose that Wnt ligands are necessary to maintain the primordial tissue of the organizer during somitogenesis by suppressing Tp53-mediated apoptosis.

Keywords: Forebrain patterning, Thalamus development, Zona limitans intrathalamica, ZLI

\section{Background}

The thalamic complex consists of the anteriorly located pre-thalamus and the posterior located thalamus [1]. The prosomeric model would describe these two areas as main dorsal components of the prosomere 3 (P3) and prosomere 2 (P2) respectively [2]. Between these two neural segments there is an intervening ventricular ridge - the zona limitans intrathalamica (ZLI). The anatomical $Z L I$ border zone contains a small cell population,

\footnotetext{
* Correspondence: steffen.scholpp@kit.edu

'Karlsruhe Institute of Technology (KIT), Institute of Toxicology and Genetics (ITG), Karlsruhe, Germany

Full list of author information is available at the end of the article
}

which releases signaling molecules. This signaling center orchestrates thalamus development by controlled release of the morphogen Sonic hedgehog and thus, we termed it the middiencephalic organizer ( $M D O$, formerly known as the ZLI organizer; [3]). Lack of the Shh-positive $M D O$ leads to gross malformation of the caudal forebrain and loss of the entire thalamus. Local abrogation of Shh signaling in small cell clones blocks acquisition of thalamic neuronal cell fate in vertebrates [4-6]. Thus the $M D O$ determines the size of the thalamic complex and orchestrates the neuronal development of the central relay station of the brain.

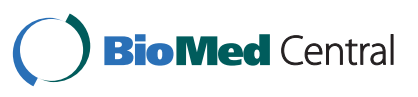

() 2012 Mattes et al; licensee BioMed Central Ltd. This is an Open Access article distributed under the terms of the Creative Commons Attribution License (http://creativecommons.org/licenses/by/2.0), which permits unrestricted use, distribution, and reproduction in any medium, provided the original work is properly cited. 
A further important diffusible, external cue during neural development is Wnt signaling. Patterning of the vertebrate anterior neural tube requires the function of this pathway at multiple stages [7]. Canonical Wnt signaling regulates anteroposterior patterning in the forebrain and midbrain, is required for development of the dorsal telencephalon [8] and the eyes [9], and allows the establishment of the midbrain-hindbrain boundary $(M H B)$ organizer [10,11]. Several lines of experimental evidence have demonstrated that other signaling pathways counteract Wnt signaling during neural development, independent from direct antagonists, such as sFRPs or Dkk1. Indeed, Shh and Wnt signaling are mutually antagonistic during some events in embryonic development, such as spinal cord patterning [12]. Despite the recognized importance of Wnt signaling for central nervous system (CNS) development, its functional relevance during diencephalon formation and how Wnt signaling and Shh signaling interact there remains unknown. Receptors, ligands and modifiers of the Wnt signaling pathway are expressed during early stages of caudal forebrain regionalization $[13,14]$. Recently, we showed that Wnt signaling is required for cell adhesion in the thalamus and thalamic neurogenesis [15]. However, the early function of Wnts during MDO establishment has to be determined.

Here we show that blockage of the canonical Wnt signaling pathway leads to malformation of the $M D O$. By a Morpholino-based knock-down approach we identified Wnt3 and Wnt3a as the responsible ligands and hence are required to maintain the primordium of the $M D O$. Lack of canonical Wnt signaling per se or knock-down of Wnt3/Wnt3a leads similarly to an increase of apoptosis specifically within the organizer primordium. Consistently, blockage of Tp53-mediated apoptosis is able to rescue the $M D O$. Furthermore, abrogation of the repressive factors Fezf2 and Irx1b leads to restoration of the organizer. In summary, we propose that canonical Wnt signaling triggered by Wnt3/Wnt3a is necessary to suppress Tp53-mediated apoptosis and thus maintain the organizer tissue during development.

\section{Results}

Several members of the Wnt family are localized at or near the roof plate - the dorsal pole of the developing nervous system where they dorsalize the embryonic brain and spinal cord [16]. In the latter, canonical Wnt signaling is opposed by the ventralizing Shh signal [17]. A similar scenario has been described for the telencephalon in which wnt8b expression is restricted by the Shh-dependent transcription factor FoxG1 [8]. To test whether canonical Wnt signaling is required for the formation of the Shh-positive $M D O$, we treated embryos with a specific Wnt signaling antagonist, the tankyrase inhibitor IWR1 [18]. We used IWR1 to block Wnt signaling from 10 to $28 \mathrm{hpf}$, after initial neural plate patterning has taken place. Under these conditions, we observed a strong reduction of Shh in the central part of the $M D O$, whereas expression at the dorsal tip remained (Figure 1A, B). Initiating treatment at $12 \mathrm{hpf}$ led to a mild decrease whereas treatment after $14 \mathrm{hpf}$ did not affect shh expression in the $M D O$ (Figure $1 \mathrm{C}, \mathrm{D}$ ), suggesting that canonical Wnt signaling is required for $M D O$ induction in a narrow time window of four hours. We used a heat-shock inducible Dkk1-GFP construct as an additional tool to inhibit Wnt signaling [19]. After activation of Dkk1-GFP at $10 \mathrm{hpf}$, we found a lack of Shh::RFP expression within the $M D O$ at $28 \mathrm{hpf}$ (Figure $1 \mathrm{E}, \mathrm{F})$. Consistently, enhancement of Wnt signaling between 10 and $28 \mathrm{hpf}$ using the GSK3ß inhibitor BIO [20] led to a broader expression domain of shh at the $M D O$ (Figure 1G). In agreement, shh expression is expanded in the diencephalon of $\operatorname{axin} 1^{-/-}$mutant embryos [21], independent of morphological alteration of the telencephalon (Figure $1 \mathrm{H}$ ). Similarly, grafting Wnt3a loaded beads in the diencephalon at $10 \mathrm{hpf}$ (Figure 1I) resulted in expansion of shh expression at the $M D O$, compared to embryos implanted with PBS-loaded control beads. Therefore, we hypothesize that canonical Wnt signaling is necessary for proper induction of the MDO between $10 \mathrm{~h}$ and $14 \mathrm{hpf}$.

Prime candidates to mediate local canonical Wnt signaling in the developing thalamus are Wnt3 and Wnt3a. At $15 \mathrm{hpf}$ and $28 \mathrm{hpf}$, both of these Wnt ligands are expressed in the $M D O$, whereas $w n t 3 a$ also marks the roof plate (Figure 2A, B). Wnt3 and Wnt3a are expressed in the mid-diencephalon prior induction of shh in the $M D O$ at $15 \mathrm{hpf}$ (Figure 2C, E). At $28 \mathrm{hpf}$, shh overlaps with wnt3 expression domain in the ventricular zone of the organizer (Figure 2F). However, expression of wnt $3 a$ becomes restricted to the dorsal MDO at 28 hpf and is subsequently localized subjacent to shh (Figure 2D). To address whether these two Wnt ligands mark the primordium of the $M D O$, we analyzed the expression pattern relative to markers in the thalamic complex. The pre-thalamic marker $\operatorname{lh} x 5$ [22] abuts the $w n t 3 a$ expression domain anteriorly (Figure 2G, H). Wnt3a overlaps with the $M D O /$ thalamus marker otx2 (I, J; [23]). The thalamic marker irx $1 b$ is expressed mainly posterior to the expression domain of wnt $3 a$ except of the most dorsal area (Figure $2 \mathrm{~K}, \mathrm{~L}$ ). Thus, wnt3 and $w n t 3 a$ mark the primordium of the MDO prior to the induction of shh expression.

To elucidate the early function of Wnt3/Wnt3a during development of the thalamic complex, we blocked their function by performing a Morpholino antisense oligomere (MO)-based knock-down approach [24]. Injection of a splice-blocking wnt3 MO leads to a severe down- 


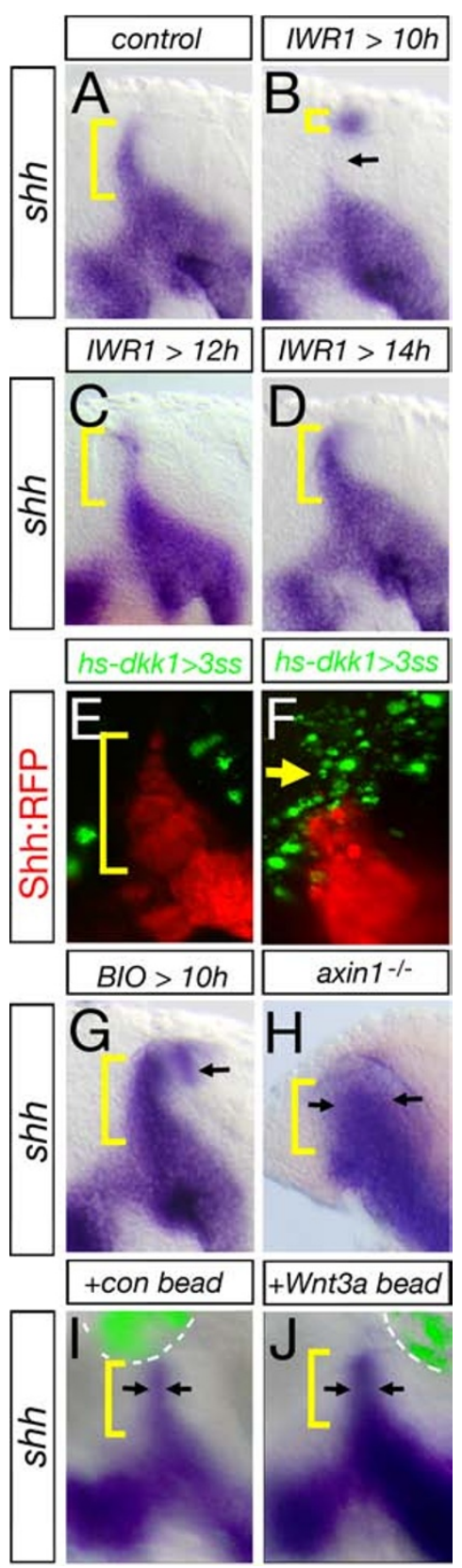

Figure 1 Canonical Wnt signaling is required for induction of Shh expression in the MDO. MD organizer in lateral views of embryos with anterior to the left at $28 \mathrm{hpf}$. Blockage of canonical Wnt signaling by treatment of embryos with $40 \mu \mathrm{M}$ IWR 1 at different time points leads to the following alterations in the formation of the shh positive MDO: 10 to $28 \mathrm{hpf}$ - strong decrease (B, arrow), 12 to $28 \mathrm{hpf}$ - mild decrease (C) 14 to $28 \mathrm{hpf}$ - no alteration (D). Shh expression at the MDO is unaltered if Dkk1-GFP positive cells are located in the telencephalon and the pretectum (E), however, the shh positive MDO is absent if Dkk1-GFP cells are located in the thalamic complex ( $F, n=6 / 10$, arrow). Ectopic activation of the Wnt signaling pathway by $\mathrm{BIO}$ treatment $(\mathrm{G}, \mathrm{n}=$ 12/18), in the axin 1-/- mutant embryos (H) as well as embryos with an implanted Wnt3a loaded bead $(I, J, n=3 / 5)$ leads to a increase of $\mathrm{MD}$ organizer shown by expanded shh expression (black arrows).

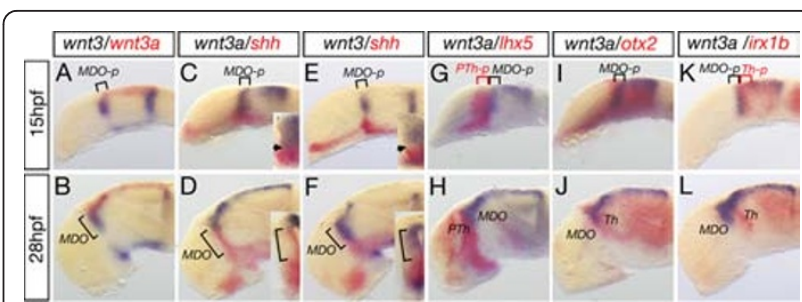

Figure 2 Wnt3 and Wnt3a mark the MDO and the dorsal thalamus. Analysis of expression dynamics of indicated marker genes at 15 and 28 hpf. At 15 hpf, wnt3 and wnt $3 a$ are expressed in the MDO primordium (MDO- $p$ ) and maintained in the organizer at 28 hpf. Cross section analysis of the left hemisphere reveals that wnt3a expression is adjacent to shh expression at the ventricular zone of the organizer (D), whereas wnt3 expression co-localizes with shh expression at $28 \mathrm{hpf}(\mathrm{F})$. In the diencephalon, expression of wnt $3 a$ is located between the $/ h \times 5$ expression domain $(G, H)$ and the irx1b expression domain ( $K, L)$. Consistently, wnt3a is coexpressed with otx2 $(\mathrm{l}, \mathrm{J})$ suggesting that wnt3a marks the $M D$ organizer territory at $15 \mathrm{hpf}$ and $28 \mathrm{hpf}$. MDO, PTh, prethalamus, Th, thalamus, *-p, *-primordium.

regulation of Wnt3 protein by Western blot analysis (Figure 3A). Brain morphology in embryos injected with either wnt3 $\mathrm{MO}$ or wnt3a $\mathrm{MO}$ is not visibly distinguishable from control MO injected embryos (Figure 3B-H). This suggests that Wnt3 and Wnt3a have redundant functions during brain development.

However, combining MOs targeting Wnt3 and Wnt3a (wnt3/wnt3a MO) led to a strong decrease of the expression of the pan- $M D O$ marker shh in the central part of the $M D O$ at 24 and 36 hpf (Figure 4A-D) These results are comparable to global Wnt signaling inhibition between 10 and $28 \mathrm{hpf}$. (Figure 1A, B). A combinatorial requirement of canonical Wnt signaling during $M H B$ organizer formation has been previously reported [11]. Indeed, the $M H B$ organizer marked by $f g f 8$ expression is lacking in double morphant embryos (Figure 4A$\mathrm{D}$, asterisks) thus validating the specificity of the wnt3/ wnt3a MO approach. In addition to the altered shh expression (also known as shh-a), we find that the expression of the paralogue $s h h-b$ is absent in the organizer in compound morphant embryos (Figure 3E, F; black arrow). Down-regulation of shh and $s h h-b$ is accompanied with a down-regulation of the Shh-dependent target gene $n k x 2.2$, (here $N k x 2.2:: G F P$ ) adjacent to the $M D O$ in wnt3/wnt3a morphant embryos (Figure 4G, $\mathrm{H})$.

Next, we analyzed the expression of components of the Wnt signaling pathway, wnt $8 b$ and axin2 in wnt3/ wnt $3 a$ morphant embryos. We observed a reduction of $w n t 8 b$ expression in the central area of the $M D O$ (Figure 4I, J). Consequently, the Wnt signaling target gene axin2 shows a reduced expression in the caudal forebrain (Figure $4 \mathrm{~K}, \mathrm{~L}$ ). These results suggest that Wnt3 and Wnt3a are required for proper $M D O$ formation. All 


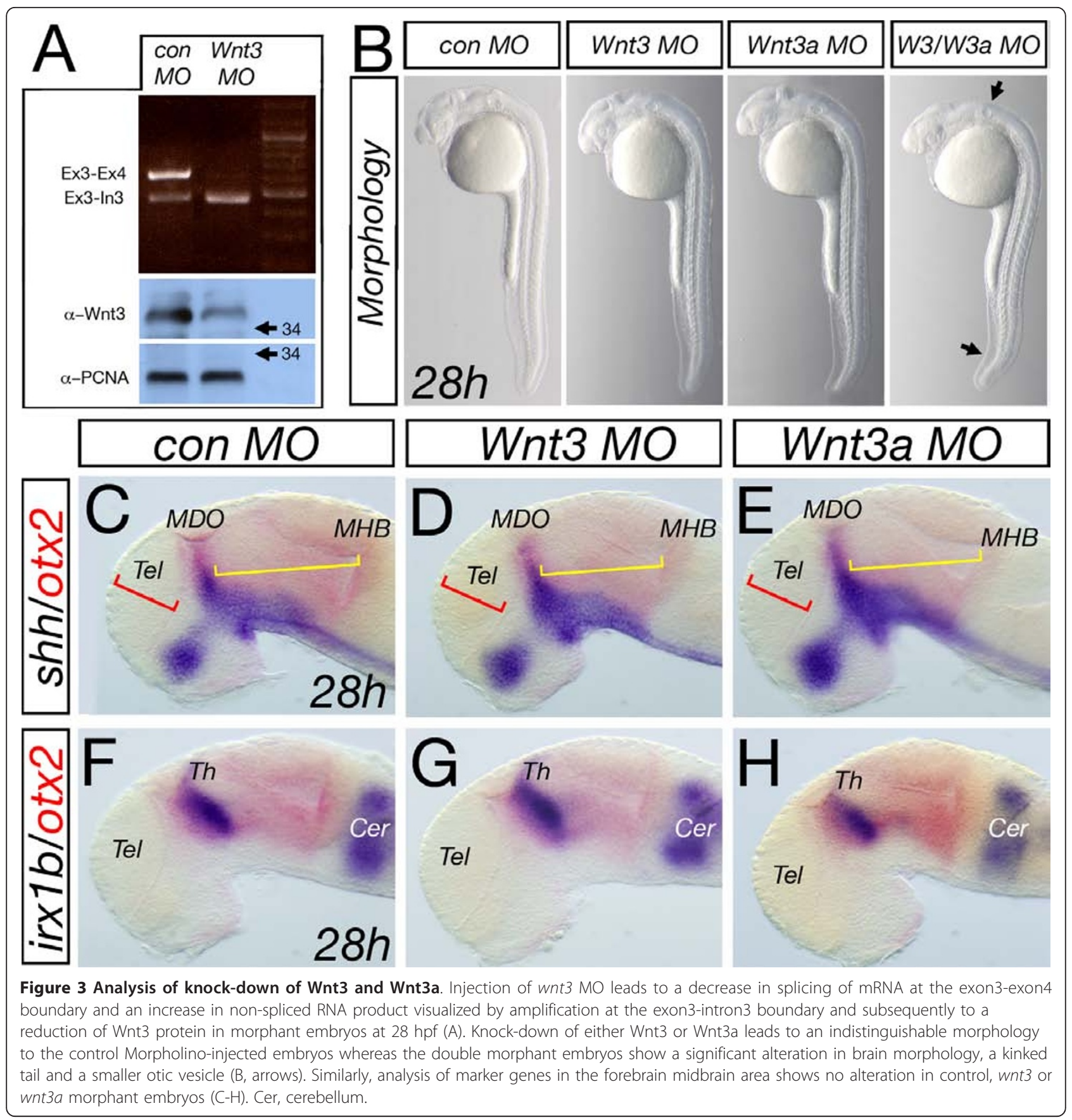

analyzed marker genes show a consistent alteration - the central area of the MDO shows a down-regulation, whereas the dorsal tip seems to be less affected by the knock-down and displays a residual robust expression of MDO markers such as shh and wnt8b (Figure 4D, H, J, $\mathrm{L})$. We wondered whether $M D O$ fate is induced within tip cells of compound morphant embryos or if cells from the basal plate migrate dorsally to form the $M D O$ spot. Therefore, we performed a time-lapse analysis using the Shh::RFP transgenic zebrafish line with strong expression of the transgene in the basal plate prior $M D O$ formation [25]. At $27 \mathrm{hpf}$, we detected Shh::RFP expression in the ventral $M D O$ with a progressively dorsal expansion over the next $12 \mathrm{~h}$ (Figure 4M). In Wnt3/ Wnt3a-deficient embryos we observed induction of dorsal MDO shh expression independently of basal plate contact (Figure $4 \mathrm{~N}$ ). This is in agreement with the socalled bucket-brigade induction model of the $M D O$ [6]. In this model cells progressively adopt $M D O$ fate from ventral to dorsal without changing their dorso-ventral 


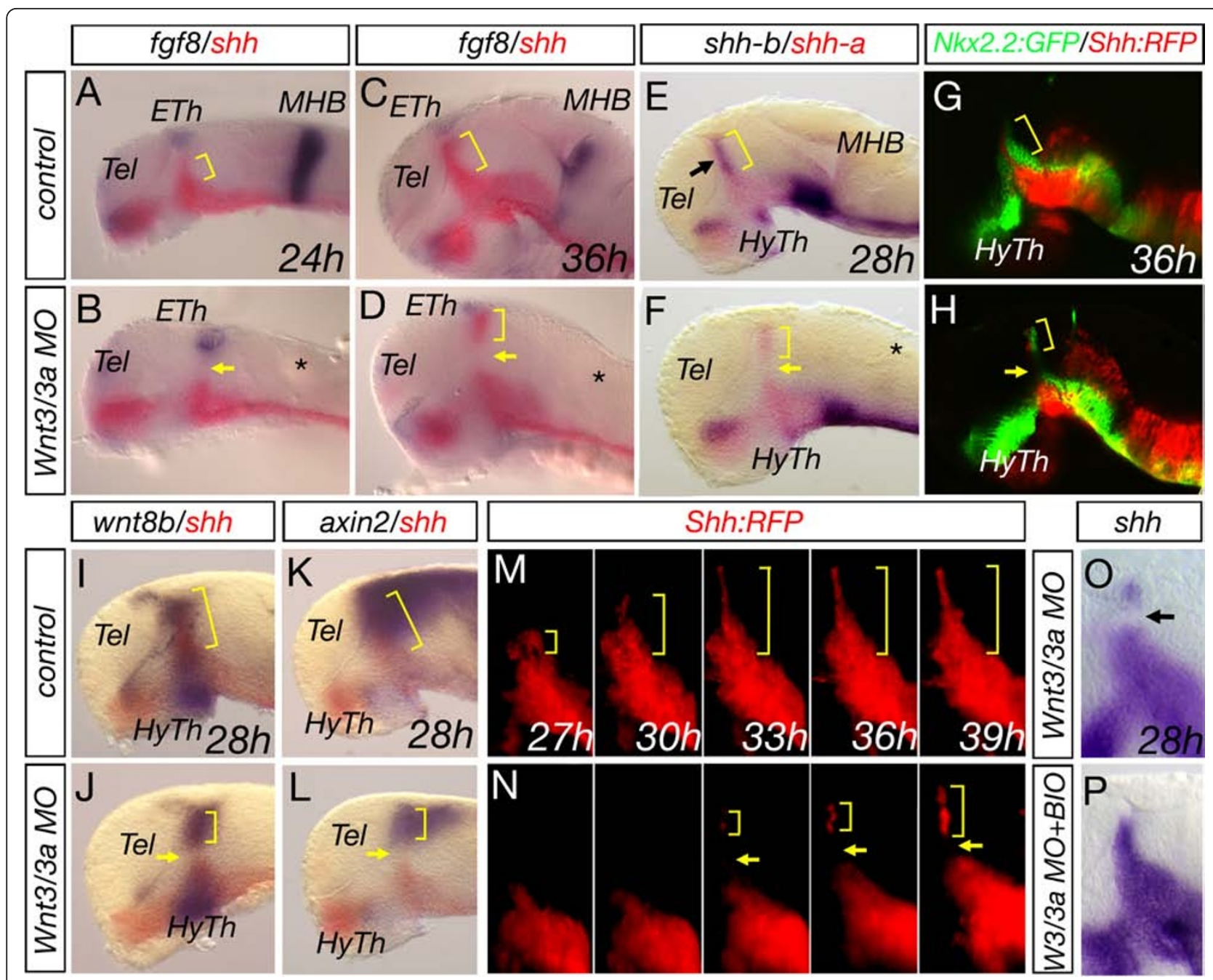

Figure 4 Wnt3 and Wnt3a are required for MDO formation. Knock-down of Wnt3 and Wnt3a leads to a down-regulation of the expression of indicated markers in the central part of the MDO (A-L). Time-lapse analysis of Shh::RFP shows an independent induction of the dorsal remaining organizer spot $(\mathrm{M}, \mathrm{N})$. Treatment of wnt3/wnt3a morphant embryos with the Wnt signaling agonist $\mathrm{BIO}$ from 10 to $28 \mathrm{~h}$ leads to a rescue of shh expression at the $M D O(O, P)$. Brackets mark the extent of the $M D O$, whereas the arrows indicate the gap between the basal plate and the remaining spot of dorsal MDO identity. Eth, epithlamus, HyTh, hypothalamus, MHB, midbrain-hindbrain boundary, Tel, telencephalon.

position. Importantly, activation of the canonical Wnt pathway using the GSK3ß inhibitor BIO between 10 and $28 \mathrm{hpf}$ is sufficient to restore $M D O$ formation in Wnt3/ Wnt3a deficient embryos (Figure 4O, P).

From the performed knock-down experiments, we conclude that canonical Wnt signaling between 10 and $14 \mathrm{hpf}$, by Wnt3/Wnt3a, is required for shh induction at the $M D O$.

Next we studied the consequences of the knock-down of Wnt3/Wnt3a on thalamic regionalization. Therefore, we analyzed the expression pattern of the prethalamic marker $f e z f 2$ and the thalamic marker $i r x 1 b$ at $15 \mathrm{hpf}$ and at 28 hpf (Figure 5A-D, 5G-J). We find that the size of expression domains of $f e z f 2$ and $i r x 1 b$ are unaltered in Wnt3/Wnt3a compound morphant embryos compared to the control embryos. In addition, in mouse and fish embryos with reduced Otx function, a similar lack of the $M D O$ and of the thalamus has been noted $[23,26]$. We find that the expression of the forebrain/ midbrain marker otx $1 l$ (Figure 5E, F, K, L) and of ot $x 2$ (Figure 6D, E') is reduced in the morphant forebrain and midbrain suggesting a similar functional connection between Otx and Shh in the compound morphant embryos.

To elucidate this aspect further, we mapped the expression of markers of the prethalamus relative to markers of the thalamus. Interestingly, we found the $l h \times 5$ positive prethalamus abuts the $i r x 1 b$ positive thalamus and the $M D O$ anlage is lacking in compound morphant embryos at $28 \mathrm{hpf}$ (Figure 6A-B'). Likewise, 


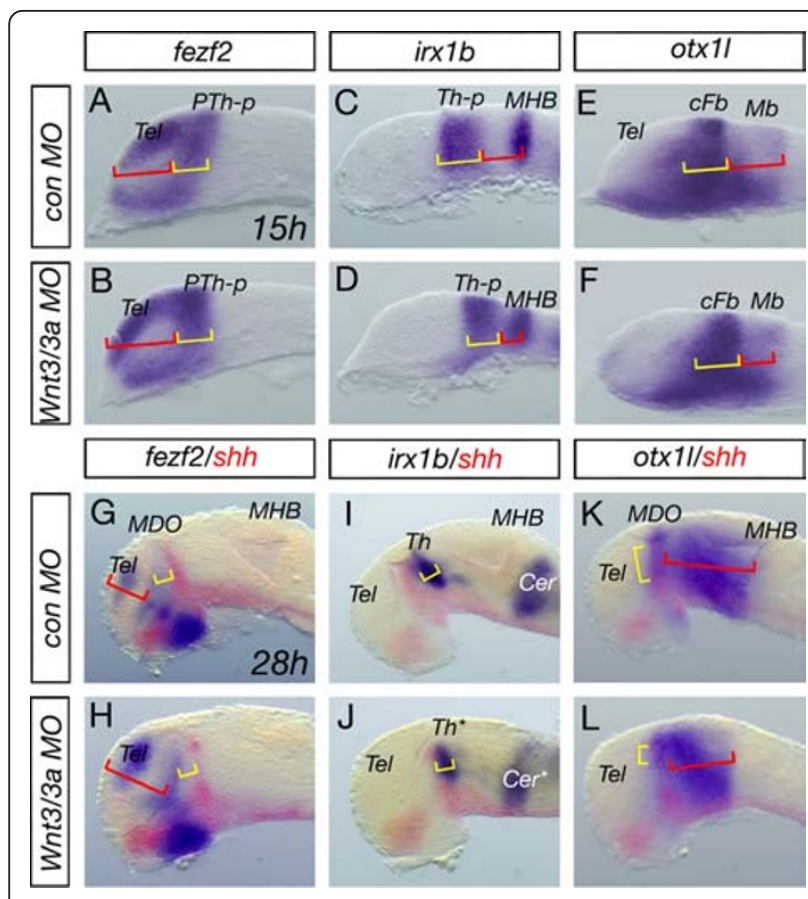

Figure 5 Primordia of the prethalamus and the thalamus are not affected in Wnt3/Wnt3a morphant embryos. Analysis of the fezf2 positive anterior forebrain area at 15 and $28 \mathrm{hpf}$ reveals an expansion of the telencephalic domain (red brackets), however, the prethalamus (yellow brackets) is unaltered wnt3/wnt3a morphant embryos (A, B, G, H). Posteriorly analysis of the irx $1 b$ as well as the otx $1 /$ expression shows that the thalamic domain does not change significantly (yellow brackets), whereas, the midbrain territory shrinks in compound morphant embryos (red brackets, C-F; I-L). Notably, although both organizers - the $M D O$ and $M H B$ - disappeared in wnt3/wnt3a morphant embryos, expression of competence factors like fezf2 and $i r \times 1 b$ are unaltered at $28 \mathrm{hpf}$, suggesting a specific function of Wnt3/Wnt3a in organizer formation. CFb, caudal forebrain, Mb, midbrain.

analysis of the $M D O$ and thalamus primordia in these embryos showed that the otx2-positive $M D O$ is severely decreased, whereas the size of the $\operatorname{irx} 1 b$ and otx2-positive thalamus is unaltered (Figure 6D-E'). This suggests that Wnt3/Wnt3a are required for establishment of the $M D O$ primordium. Consistently, blockage of Wnt signaling with IWR1 between 10 and $28 \mathrm{hpf}$ leads to a similar phenotype (Figure 6C, C', F, F'). This suggests that Wnt3/Wnt3a function is required to maintain the anlage of the $M D O$, but not for maintenance of the primordia of the prethalamus and thalamus.

We next considered the Wnt-mediated process that maintains the $M D O$ anlage. Wnt signaling plays a pivotal role in cell survival [27] providing a possible explanation for the lack of the MDO primordium in Wnt3/ Wnt3a deficient embryos. Therefore, we analyzed apoptosis by usage of the fluorescent cationic dye, acridine orange, which permeates dying cells to bind chromatin [28]. Indeed, compound morphant embryos display an

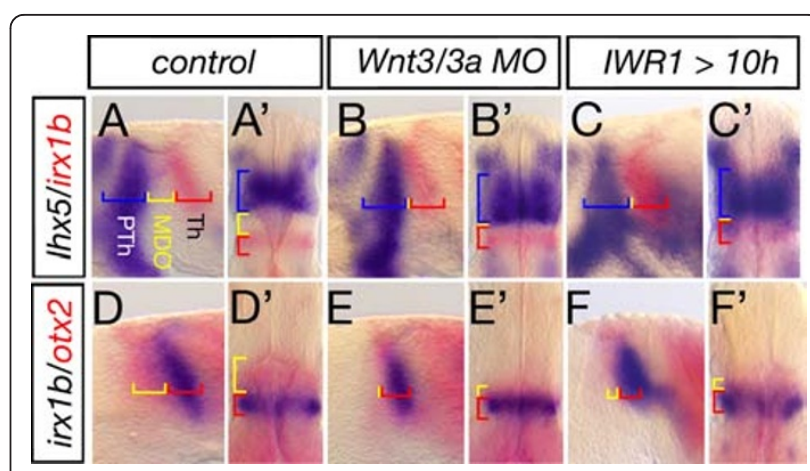

Figure 6 Blockage of Wnt signaling lead to lack of MDO tissue. Lateral views and dorsal views (marked by ') of embryonic heads at 28 hpf. Wnt3/wnt3a morphant embryos lack the organizer tissue and the 1 h $\times 5$ positive prethalamus (PTh, blue) abuts the ir $1 \mathrm{~b}$ thalamus (Th, red) $\left(A-B^{\prime}, n=54 / 78\right)$. A similar phenotype is observed in embryos treated with $30 \mu \mathrm{M}$ IWR1 from 10 to $28 \mathrm{~h}$ to inhibit canonical Wht signaling $(n=15 / 17)$. Hence, the otx2 positive MDO (yellow) is lacking if Wnt3/Wnt3a function is knocked-down ( $n=44$ / 80, D-E'), likewise after inhibition of canonical Wnt signaling ( $F, F^{\prime}, n$ $=14 / 24)$.

accumulation of dying cells within the organizer primordium (arrow, Figure 7A-B'). Consistently, blockage of canonical Wnt signaling by treatment with IWR1 leads to a similar increase of apoptotic cells specifically within the organizer anlage (Figure $\left.7 \mathrm{C}, \mathrm{C}^{\prime}\right)$. Consistently, blockage of Tp53 function is able to rescue shh expression in the organizer in Wnt3/Wnt3a morphant embryos as well as in IWR1 treated embryos (Figure 7D-F). This suggests that a main function of Wnt3/Wnt3a in the caudal forebrain is to ensure the survival of $M D$ organizer cells.

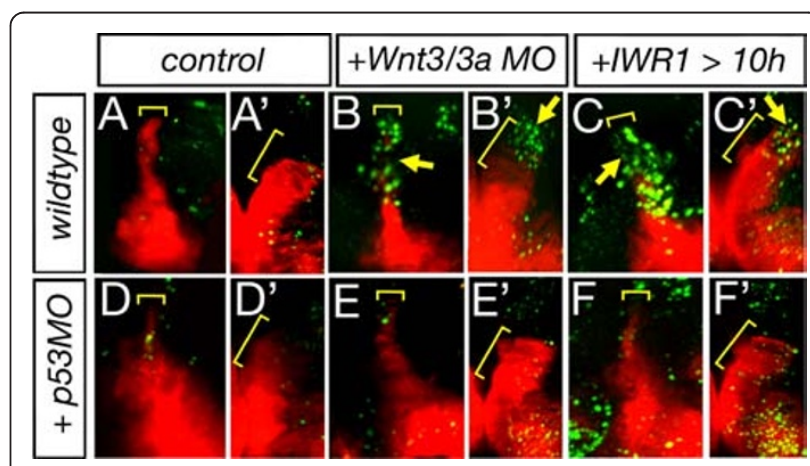

Figure 7 Lack of Wnt signaling leads to apoptosis in the MDO Lateral views and dorsal views of the left hemisphere (marked by ') of Shh::RFP transgenic embryos at 36 hpf. Wnt3/wnt3a morphant embryos as well as embryos treated with IWR1 display an increase of apoptotic cells within the MDO at $36 \mathrm{hpf}\left(\mathrm{n}=4 / 6, \mathrm{~A}-\mathrm{C}^{\prime}\right)$. Upon blockage of Tp53 function apoptosis is down-regulated within the organizer tissue of wnt3/wnt3a morphant embryos as well as embryos treated with IWR1 and consistently, shh:RFP expression is restored $\left(D-F^{\prime}\right)$. 
Previous evidence has suggested that members of two transcription factor families, Fez [29] and Irx [30] are involved in induction of the Shh-positive organizer. However, recent observations show that prethalamic Fez limits the anterior border of the $M D O$ [31], whereas the thalamic Irx genes are able to repress Shh expression at the posterior limit of the $M D O[1,23]$. Therefore, we asked if the main function of Wnt3/Wnt3a in the developing thalamus is the establishment of a Fez/Irx-free zone in the caudal forebrain to allow formation of the MDO. We first tested whether in this situation a similar reduction of $s h h$ is seen in embryos deficient for Wnt3/ Wnt3a function (Figure 8A, B). As a broadening of the $M D O$ in embryos deficient for FezF2 [31] and Irx1b [23] has been reported previously, we wondered if we would be able to restore the $M D O$ in the individual triple morphant embryos. Indeed, upon simultaneous reduction of Fezf2 or Irx1b function, we observe a rescue of shh expression at the $M D O$ of wnt $3 / w n t 3 a$ morphant embryos (Figure 8C, D). To elucidate the impact of the knock-down on the formation of the thalamic complex, we analyzed the expression of the pre-thalamic marker lhx 5 and the thalamic proneural factor neurog1. In $w n t 3 / w n t 3 a / f e z f 2$ triple morphant embryos, the broadening of the shh-positive $M D O$ is accompanied with down-regulation of the dorsal pre-thalamic $l h \times 5+$ domain, suggesting that the $M D O$ expands at the

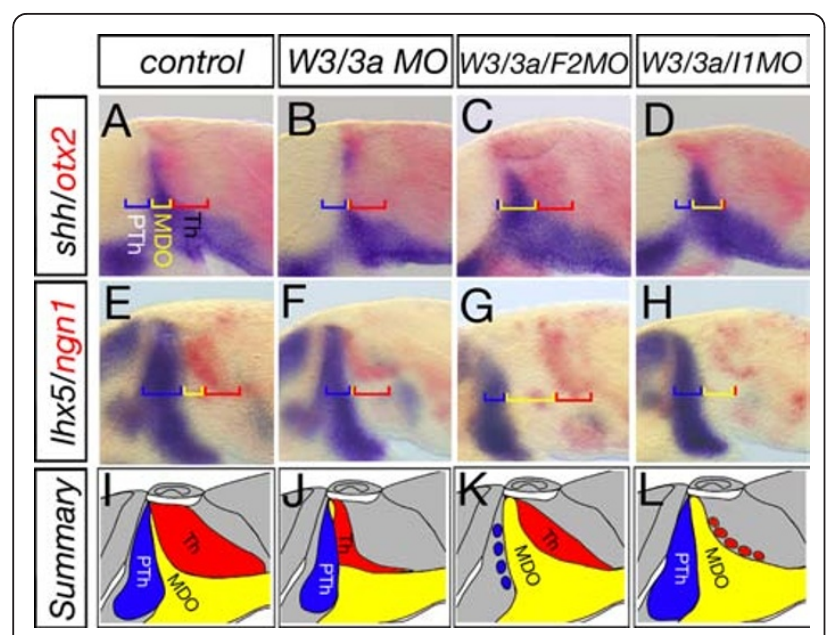

Figure 8 FezF2 and Irx1b limit the MDO at the AP axis. Shh expression in the $M D O$ is decreased in Wnt3/Wnt3a deficient embryos ( $A, B, n=45 / 55)$, whereas, prethalamic identity as well as thalamic identity is maintained ( $E, F, n=18 / 23)$. In triple morphant embryos lacking Wnt3, Wnt3a and Fezf2 function, a restoration of shh expression is observed in the $M D O(C, n=28 / 31)$ accompanied by a reduction of $/ h \times 5$ expression in the pre-thalamus ( $G, n=10$ / 34). In wnt3/wnt3a/irx1b triple morphant embryos, we observe a similar restore of the shh positive $M D O(D, n=28 / 31)$; however, here the neurogl positive thalamus shrinks ( $H, n=25 / 32, N, n=20$ / 25). For explanation of the summary (I-L) see the text. expense of the pre-thalamus (Figure 8G). On the other hand, the shh expression domain at the $M D O$ that is restored in the wnt3/wnt3a/irx $1 b$ morphant embryos is accompanied by a reduction of the neurog 1 positive thalamus, presumably by expansion of the $M D O$ into the thalamic territory (Figure $8 \mathrm{H}$ ). We, therefore, conclude that both adjacent areas - prethalamus and thalamus would be competent to act as MDO. However, Fez and Irx are required to repress organizer formation, to shape the overall domain of the $M D O$ and to maintain the identities of adjacent brain regions (Figure 8I-L).

\section{Discussion}

Here we have described a new aspect of Wnt signaling during caudal forebrain development. Wnt3 and Wnt3a mark the $M D O$ prior to the expression of the principal thalamus organizing signal Shh. Blockade of Wnt signaling leads to the lack of the $M D O$ tissue (Figure 9). We show that cells of the organizer primordium go into Tp53-mediated apoptosis upon loss of the Wnt stimulus. We hypothesize that the main function of Wnt3/ Wnt3a-mediated signaling in the organizer is to protect cells from cell death as blockage of Tp53-mediated apoptosis is able to restore the organizer function. Thus, Wnt3/Wnt3a-mediated signaling is the survival factor for organizer cells only, but not for the surrounding thalamic complex.

\section{Wnt3 and Wnt3a are the principal, but not sole Wnt ligands during MDO formation}

In Wnt signaling-deficient embryos, we find a persistent spot of Shh-positive cells in the dorsal most tip of the organizer. There are two possibilities to explain this

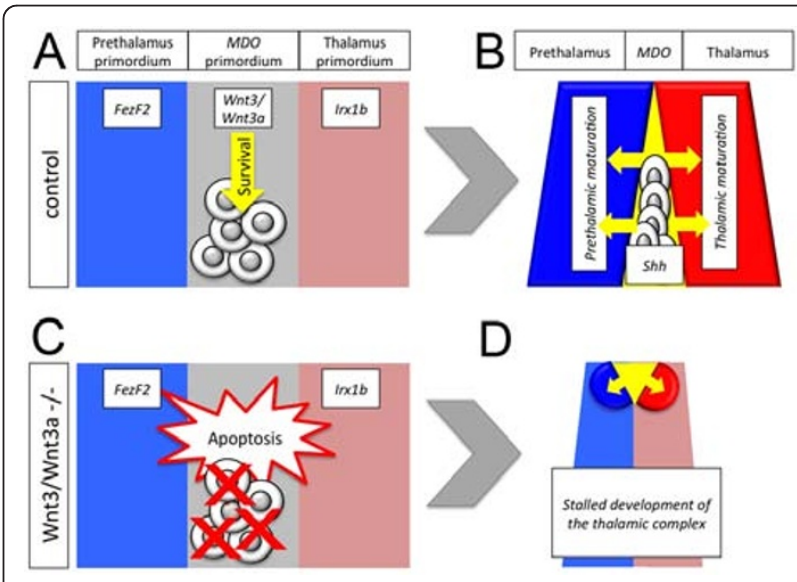

Figure 9 Summary. Wnt3 and Wnt3a signaling maintains the MDO primordium by protecting the cells from apoptosis (A). The mature MDO secrets organizer signals, such as Shh, which drives thalamic development (B). In Wnt3/Wn3a deficient embryos, MDO cells go into apoptosis (D). Loss of the organizer leads to stalled development of the thalamic complex (D). 
phenotype. First, the dorsal diencephalic roof plate is a rich source of several Wnt ligands: in addition to Wnt3a, we find expression of Wnt8b, Wnt1 and others suggesting that there is a compensation mechanism operating at the dorsal $M D O$. Indeed, in a few embryos treated with IWR1 or overexpressing the Wnt antagonist Dkk1 (Figure 1), we observed a total block of organizer formation. However, these treatments also led to gross malformation of the embryo making it difficult to identify a specific Wnt-related function. A further explanation could be the third signaling pathway important for thalamus formation, the Fgf pathway. Fgf ligands, such as Fgf8, are strongly expressed at the dorsal area of thalamic anlage - in the epithalamus. Here, Fgf signaling is required for the formation of the rostral thalamus and influences expression of thalamic transcription factors such as Gbx2 [32,33]. This could suggest that Fgf signaling is required independently to maintain $M D O$ fate, a possibility that requires future analysis.

\section{Wnt signaling during thalamus development}

Wnt signaling is important to set up the initial anteroposterior pattern of the entire neuraxis. Subsequently, Wnt signaling becomes important in individual brain regions. In the caudal forebrain, the thalamus is an area that shows enriched expression of ligands, receptors and mediators of the canonical Wnt signaling pathway. Wnt3 and Wnt3a mark the MDO and the dorsal part of the thalamus in fish, an expression pattern that is conserved in the vertebrate lineage as recent work has demonstrated that both ligands are similarly expressed in the embryonic thalamus of the chick [14] and mouse [34]. During thalamic complex development, however, a comprehensive picture of the function of Wnt signaling is still lacking and only recently individual aspects have begun to be elucidated. Inhibition of canonical Wnt signaling by Dkk-1 transforms the thalamus into pre-thalamus during the early regionalization phase [35]. Furthermore, it has been shown that the pre-thalamus marker Lhx5 can activate the expression of the extracellular Wnt inhibitor sFRP1a and sFRP5 [22]. These data suggest that canonical Wnt signaling is required for thalamus development, whereas the development of the pre-thalamus requires inhibition of canonical Wnt signaling.

The canonical Wnt signaling pathway plays a pivotal role in mediating the clustering of cells. The key effector of the Wnt pathway, $\beta$-catenin, promotes adhesiveness by binding to the transmembrane adhesion molecule cadherin [36,37]. Recently, a member of this group, the Protocadherin 10b (Pcdh10b, formerly known as OLprotocadherin) has been shown to modulate cell adhesion in the thalamic complex [15]. Stabilization of $ß$ catenin leads to a broadening of the expression domain of $p c d h 10 b$ whereas inhibition of Wnt signaling blocks $p c d h 10 b$ expression. Hence, alteration of $p c d h 10 b$ expression in the thalamus leads to an intermingling of thalamic cells with the neighboring brain areas, predominantly with the pretectum. Furthermore, Wnt signaling seems to play a crucial role in thalamic neurogenesis as post-mitotic neurons express Wnt specific target genes such as lef1 [38] and these markers have been shown to be activated by Wnt signaling during late thalamic maturation [15].

The foregoing descriptions notwithstanding, our knowledge of the requirement for Wnt signaling for the formation of the $M D O$ is still fragmented. Reduced Wnt signaling activity in the Lrp6 -/- knockout mouse led to a reduction of the $M D O$ and thalamus [39], and the expression of thalamic transcription factors, such as Gbx2, is severely down-regulated in these mice, suggesting that Lrp6-mediated Wnt signaling is required for proper thalamus development. However, organizer markers, such as Wnt3 and Shh, are similarly down-regulated. These data support our observation that lack of Wnt signaling leads to a malformation of the organizer tissue in zebrafish. Interestingly, we identified a narrow window of four hours during somitogenesis, which is sufficient to maintain the organizer anlage in zebrafish. This time point correlates with the expression dynamic of both ligands as co-expression of Wnt3 and Wnt3a in the organizer can only be observed between the 10 and the 16 somite stages (Figure 2). In light of our data the defects observed in the Lrp6-/- mouse thalamus may be interpreted as a dual phenotype, (i) disruption of the $M D O$ and (ii) misspecification of thalamic cells - both due to a lack of Wnt signaling.

\section{Fez and Irx are able to suppress MDO competence in pre- thalamus and thalamus}

Interestingly, we find that the expression of pre-thalamic markers, such as fezf2 (Figure 5) and $l h x 5$ (Figure 6), as well as thalamic markers, such as $i r x 1 b$ and otx2 (Figure 6), are not affected by the abrogation of Wnt signaling during somitogenesis. Although the $M D O$ area disappears, the size of the surrounding territories is maintained. This suggests that development of the primordia of pre-thalamus, $M D O$ and thalamus are largely independent at this early stage. Indeed, cell lineage restriction operates at the borders of the organizer $[13,40]$. However, by simultaneous knockdown of Fezf2 function in the pre-thalamus or Irx1b function in the thalamus in Wnt deficient embryos, we were able to rescue the formation of the organizer. We found that the territory of the caudal forebrain and midbrain is similarly small in Wnt3/Wnt3a double morphant embryos compared to the triple morphant embryos. Therefore, we propose a comparable increased rate of apoptosis. However, we 
found that both pre-thalamus and thalamus are competent to form the MDO organizer. However, they lose their competence for organizer induction by expression of the transcription factors, Fez or Irx. Indeed, both transcription factors have been characterized by their pivotal repressive function during neural development [41-43]. For example, Irx2 restricts the $M H B$ organizer primordium by suppression of the competence in the cerebellum to adopt $M H B$ organizer fate [42]. Consequently, a dominant-negative version of Irx2 leads to ectopic induction of the organizer. Although the $M H B$ organizer is characterized by the expression of several Wnt ligands, the relation between Wnt signaling and Irx function is unclear during organizer formation.

Thus, we may conclude that canonical Wnt signaling is required for maintenance of the organizer primordium and, subsequently, for the formation of the entire thalamic complex.

\section{Wnt signaling and apoptosis}

Wnt signaling has been suggested as a crucial survival factor in many contexts. In Drosophila, patches of cells that are deficient in Wg signal transduction are progressively eliminated by apoptosis $[44,45]$. In vertebrates, Wnt signaling has been suggested as an important external trigger for proliferation of stem cell and cancer cells [27]. During the development of the central nervous system, stabilization of $ß$-catenin in neural precursors leads to enlarged brains with increased cerebral cortical surface area and folds, suggesting that Wnt signaling can regulate cerebral cortical size by controlling the generation of neural precursor cells [46]. Consistently, reduction of $B$-catenin signaling leads to reduction of central nervous tissue as the neuronal precursor population is not maintained [47]. Here, we show that blockade of canonical Wnt signaling leads to specific cell death in the $M D O$. Recently, it has been suggested that Morpholino oligomeres per se may induce Tp53mediated apoptosis $[48,49]$. However, we provide evidence that apoptosis observed in compound morphant embryos is due to a specific loss of Wnt3/Wnt3a function. First, we observed locally enriched apoptosis within the organizer tissue, but the surrounding areas are unaffected. Second, we observed a similar apoptotic phenotype after treatment with the small molecule inhibitor IWR1 and the organizer is similarly reduced in embryos with ectopically induced Dkk1 expression. Third, we were able to restore the organizer in double morphant embryos by treatment with the Wnt agonist $\mathrm{BIO}$ and we rescue the organizer in embryos treated with IWR1 by simultaneously blocking Tp53-mediated apoptosis. Taking these arguments together, we conclude that Wnt3 and Wnt3a are required for protecting the organizer tissue from Tp53-mediated apoptosis. Consistently, our findings are supported by a recent observation in cancer cells suggesting that Tp53-mediated apoptosis acts in a negative feedback loop with Wnt signaling [50].

\section{Conclusion}

In summary, we show that canonical Wnt signaling is required for regionalization of the caudal forebrain. Alteration of the canonical Wnt signaling pathway leads to apoptosis of the $M D O$ primordium and subsequently to a mis-specification of the entire thalamic complex (Figure 9). We suggest that Wnt3 and Wnt3a are the crucial Wnt ligands, which are required between $10 \mathrm{~h}$ and $14 \mathrm{~h}$ to maintain the $M D O$ anlage by protecting the cells from Tp53mediated apoptosis. Thus, by determining $M D O$ fate and thalamic compartition, Wnt3 and Wnt3a control the development of the organizer of the major relay station in the brain - the thalamus.

\section{Methods}

\section{Maintenance of fish}

Breeding zebrafish (Danio rerio) were maintained at $28^{\circ}$ $\mathrm{C}$ on a $14 \mathrm{~h}$ light/10 h dark cycle [51]. To prevent pigment formation, embryos were raised in $0.2 \mathrm{mM} 1$-phenyl-2-thiourea (PTU, Sigma, St. Louis, MO 63103 USA) after $24 \mathrm{hpf}$. The data we present in this study were acquired from analysis of KIT wild-type zebrafish $\mathrm{AB} 2 \mathrm{O} 2$ as well as the transgenic zebrafish line Shh::RFP [25], Nkx2.2::GFP and masterblind mutant line carrying a mutation in $\operatorname{axin1}$ [21].

\section{Functional analysis}

Transient knock-down of gene expression was performed as described in [52]. We used the following Morpholino-antisense oligomeres (MO, Gene Tools, Philomath, OR 97370 USA) at a concentration of 0.5 mM: wnt3 MO (5'-GATCTCTTACCATTCGTCCTGC3'), $0.25 \mathrm{mM}$ wnt3a MO [53], irx1b MO [54], fezf2 MO [31], and Tp53 MO [55]. The injection of MO oligomers was performed into the yolk cell close to blastomeres at one-cell or two-cell stage.

To manipulate Wnt signaling in-vivo, we used BIO [20]; (2'Z,3'E)-6-Bromo-indirubin-3'oxime, TOCRIS Bioscience, Minneapolis, MN 55413 USA) or IWR-1 [18]; (Sigma) as pharmacological agonist and antagonist of the Wnt signaling pathway. For Wnt signaling analyzes, embryos were dechorionated and incubated with 4 $\mu \mathrm{M}$ of $\mathrm{BIO}$ in $1 \%$ dimethyl sulfoxide (DMSO), $40 \mu \mathrm{M}$ IWR-1 in $0.2 \%$ DMSO or with $1 \%$ DMSO only at given time points. Heparin-coated acrylic beads (Adar Biotech, Rehovot 76360 Israel) were prepared as described previously [56]. The beads were coated with recombinant Wnt3a protein (R\&D Systems, Minneapolis, MN 55413 USA) and implanted dorsally into the region of the presumptive $M D O$ of wild-type embryos at the $10 \mathrm{hpf}$. HSDkk1-GFP DNA [57] was injected into one-cell stage 
embryos. A 15 -minute heat shock treatment at $42^{\circ} \mathrm{C}$ was performed at $10 \mathrm{hpf}$. All treated embryos were incubated at $28^{\circ} \mathrm{C}$ and fixed at $28 \mathrm{hpf}$.

\section{Staining procedures}

Prior to staining, embryos were fixed in $4 \%$ paraformaldehyde/PBS at $4^{\circ} \mathrm{C}$ overnight for further analysis. Whole-mount mRNA in situ hybridizations were performed as described in [58]. The expression pattern and/or antisene RNA probes have been described for wnt3 (formerly known as wnt3l) and wnt3a [59], shh (shh-a) [60]), shh-b [61], ptc1 [62], axin2 [63], lhx5 [64], irx1b [54], otx1l and otx2 [65], neurog1 [66], fezf2 [67].

SDS-PAGE/Western blot analysis was performed with polyclonal antibodies to detect Wnt3 (GTX105679, Acris Antibodies, San Diego, CA 92121 USA) and Wnt3a (ab28472, Abcam, Cambridge, CB4 OFL UK) and a monoclonal antibody against PCNA (sc-56, Santa Cruz Biotechnology, Santa Cruz, CA, USA) as loading control, respectively.

\section{Image acquisition}

Prior to imaging, embryos were de-yolked, dissected and mounted in $70 \%(\mathrm{v} / \mathrm{v})$ glycerol/PBS on slides with cover slips. Images were taken on an Olympus SZX16 microscope equipped with a DP71 digital camera by using the imaging software Cell A. For confocal analysis, embryos were embedded for live imaging in $1.5 \%$ low-meltingpoint agarose (Sigma) dissolved in $1 \times$ Ringer's solution containing $0.016 \%$ tricaine at $48 \mathrm{hpf}$. Confocal images stacks were obtained using the Leica TCS SP5 confocal laser-scanning microscope. We collected a series of optical planes (z-stacks) to reconstruct the imaged area. Rendering the volume in three dimensions provided a view of the image stack at different angles. The step size for the $\mathrm{z}$-stack was usually 1 to $2 \mu \mathrm{m}$ and was chosen upon calculation of the theoretical z-resolution of the $40 \times$ objective. Images were further processed using Imaris 6 (Bitplane AG, CH-8048, Zurich Switzerland).

\section{Abbreviations \\ Cer: Cerebellum; cFb: Caudal forebrain; DMSO: Dimethyl sulfoxide; Eth: Epithlamus; HyTh: Hypothalamus; Mb: Midbrain; MDO: Mid-diencephalic organizer; MHB: Midbrain-hindbrain boundary; PBS: Phosphate-buffered saline; PTh: Pre-thalamus; PTh-p: Pre-thalamus primordium; Tel: Telencephalon; Th: Thalamus; Th-p: Thalamus primordium; ZLI: Zona limitans intrathalamica.}

\section{Acknowledgements}

We would like to thank Daniela Peukert for technical help and Andrew Lumsden (MRC Centre of Developmental Neurobiology, King's College London) for critical reading of the manuscript. BM, SW, DP and SS are funded by the Emmy-Noether grant $847 / 2$ of the Deutsche

Forschungsgemeinschaft (DFG). The Medical Research Council, UK, supports JP and $\mathrm{CH}$.

\section{Author details}

${ }^{1}$ Karlsruhe Institute of Technology (KIT), Institute of Toxicology and Genetics (ITG), Karlsruhe, Germany. ${ }^{2}$ MRC Centre for Developmental Neurobiology, King's College London, London, UK.

\section{Authors' contributions}

BM, SW and JP carried out the molecular genetic studies displayed in this manuscript. QC carried out the immunoassays. SS, CH and GD conceived the study, and participated in its design and coordination. SS guided the project and wrote the manuscript. All authors read and approved the final manuscript.

\section{Competing interests}

The authors declare that they have no competing interests.

Received: 27 January 2012 Accepted: 4 April 2012

Published: 4 April 2012

\section{References}

1. Kiecker C, Lumsden A: Compartments and their boundaries in vertebrate brain development. Nat Rev Neurosci 2005, 6:553-564.

2. Puelles $L$, Rubenstein $J$ : Forebrain gene expression domains and the evolving prosomeric model. Trends Neurosci 2003, 26:469-476.

3. Scholpp S, Lumsden A: Building a bridal chamber: development of the thalamus. Trends Neurosci 2010, 33:373-380.

4. Kiecker $C$, Lumsden A: Hedgehog signaling from the $Z$ LI regulates diencephalic regional identity. Nat Neurosci 2004, 7:1242-1249.

5. Vieira C, Garda AL, Shimamura K, Martinez S: Thalamic development induced by Shh in the chick embryo. Dev Biol 2005, 284:351-363.

6. Scholpp S, Wolf O, Brand M, Lumsden A: Hedgehog signaling from the zona limitans intrathalamica orchestrates patterning of the zebrafish diencephalon. Development 2006, 133:855-864.

7. Wilson SW, Houart C: Early steps in the development of the forebrain. Dev Cell 2004, 6:167-181.

8. Danesin C, Peres JN, Johansson M, Snowden V, Cording A, Papalopulu N, Houart C: Integration of telencephalic Wnt and hedgehog signaling center activities by Foxg1. Dev Cell 2009, 16:576-587.

9. de longh RU, Abud HE, Hime GR: WNT/Frizzled signaling in eye development and disease. Front Biosci 2006, 11:2442-2464.

10. McMahon AP, Bradley A: The Wnt-1 (int-1) proto-oncogene is required for development of a large region of the mouse brain. Cell 1990, 62:1073-1085.

11. Lekven AC, Buckles GR, Kostakis N, Moon RT: Wnt1 and wnt10b function redundantly at the zebrafish midbrain-hindbrain boundary. Dev Biol 2003, 254:172-187

12. Altmann $\mathrm{CR}$, Brivanlou $\mathrm{AH}$ : Neural patterning in the vertebrate embryo. Int Rev Cytol 2001, 203:447-482.

13. Garcia-Lopez R, Vieira C, Echevarria D, Martinez S: Fate map of the diencephalon and the zona limitans at the 10-somites stage in chick embryos. Dev Biol 2004, 268:514-530.

14. Quinlan R, Graf M, Mason I, Lumsden A, Kiecker C: Complex and dynamic patterns of Wnt pathway gene expression in the developing chick forebrain. Neural Dev 2009, 4:35.

15. Peukert D, Weber S, Lumsden A, Scholpp S: Lhx2 and Lhx9 determine neuronal differentiation and compartition in the caudal forebrain by regulating Wnt signaling. PLOS Biol 2011, 9:e1001218.

16. Alvarez-Medina R, Cayuso J, Okubo T, Takada S, Marti E: Wnt canonical pathway restricts graded Shh/Gli patterning activity through the regulation of Gli3 expression. Development 2008, 135:237-247.

17. Sousa VH, Fishell $\mathrm{G}$ : Sonic hedgehog functions through dynamic changes in temporal competence in the developing forebrain. Curr Opin Genet Dev 2010, 20:391-399.

18. Huang SM, Mishina YM, Liu S, Cheung A, Stegmeier F, Michaud GA, Charlat O, Wiellette E, Zhang Y, Wiessner S, Hild M, Shi X, Wilson CJ, Mickanin C, Myer V, Fazal A, Tomlinson R, Serluca F, Shao W, Cheng H, Shultz M, Rau C, Schirle M, Schlegl J, Ghidelli S, Fawell S, Lu C, Curtis D, Kirschner MW, Lengauer $C$, et al: Tankyrase inhibition stabilizes axin and antagonizes Wnt signaling. Nature 2009, 461:614-620.

19. Caneparo L, Huang YL, Staudt N, Tada M, Ahrendt R, Kazanskaya O, Niehrs C, Houart C: Dickkopf-1 regulates gastrulation movements by coordinated modulation of Wnt/beta catenin and Wnt/PCP activities, through interaction with the Dally-like homolog Knypek. Genes Dev 2007, 21:465-480.

20. Meijer L, Skaltsounis AL, Magiatis P, Polychronopoulos P, Knockaert M, Leost M, Ryan XP, Vonica CA, Brivanlou A, Dajani R, Crovace C, Tarricone C, 
Musacchio A, Roe SM, Pearl L, Greengard P: GSK-3-selective inhibitors derived from Tyrian purple indirubins. Chem Biol 2003, 10:1255-1266.

21. Heisenberg CP, Houart C, Take-Uchi M, Rauch GJ, Young N, Coutinho P, Masai I, Caneparo L, Concha ML, Geisler R, Dale TC, Wilson SW, Stemple DL: A mutation in the Gsk3-binding domain of zebrafish Masterblind/Axin1 leads to a fate transformation of telencephalon and eyes to diencephalon. Genes Dev 2001, 15:1427-1434.

22. Peng G, Westerfield M: Lhx5 promotes forebrain development and activates transcription of secreted Wnt antagonists. Development 2006, 133:3191-3200

23. Scholpp S, Foucher I, Staudt N, Peukert D, Lumsden A, Houart C: Otx11, Otx2 and Irx1b establish and position the ZLI in the diencephalon. Development 2007, 134:3167-3176.

24. Nasevicius A, Ekker SC: Effective targeted gene 'knockdown' in zebrafish. Nat Genet 2000, 26:216-220.

25. Peravali R, Gehrig J, Giselbrecht S, Lutjohann DS, Hadzhiev Y, Muller F, Liebel U: Automated feature detection and imaging for high-resolution screening of zebrafish embryos. Biotechniques 2011, 50:319-324.

26. Acampora D, Avantaggiato V, Tuorto F, Simeone A: Genetic control of brain morphogenesis through Otx gene dosage requirement. Development 1997, 124:3639-3650

27. Reya T, Clevers H: Wnt signaling in stem cells and cancer. Nature 2005, 434:843-850.

28. Tucker B, Lardelli M: A rapid apoptosis assay measuring relative acridine orange fluorescence in zebrafish embryos. Zebrafish 2007, 4:113-116.

29. Hirata T, Nakazawa M, Muraoka O, Nakayama R, Suda Y, Hibi M: Zinc-finger genes Fez and Fez-like function in the establishment of diencephalon subdivisions. Development 2006, 133:3993-4004.

30. Kobayashi D, Kobayashi M, Matsumoto K, Ogura T, Nakafuku M, Shimamura K: Early subdivisions in the neural plate define distinct competence for inductive signals. Development 2002, 129:83-93.

31. Jeong JY, Einhorn Z, Mathur P, Chen L, Lee S, Kawakami K, Guo S: Patterning the zebrafish diencephalon by the conserved zinc-finger protein Fezl. Development 2007, 134:127-136.

32. Kataoka A, Shimogori T: Fgf8 controls regional identity in the developing thalamus. Development 2008, 135:2873-2881.

33. Martinez-Ferre A, Martinez S: The development of the thalamic motor learning area is regulated by Fgf8 expression. J Neurosci 2009, 29:13389-13400.

34. Bluske KK, Kawakami Y, Koyano-Nakagawa N, Nakagawa Y: Differential activity of Wnt/beta-catenin signaling in the embryonic mouse thalamus. Dev Dyn 2009, 238:3297-3309.

35. Braun MM, Etheridge A, Bernard A, Robertson CP, Roelink H: Wnt signaling is required at distinct stages of development for the induction of the posterior forebrain. Development 2003, 130:5579-5587.

36. Huber O, Bierkamp C, Kemler R: Cadherins and catenins in development. Curr Opin Cell Biol 1996, 8:685-691.

37. Bienz M: beta-Catenin: a pivot between cell adhesion and Wnt signaling. Curr Biol 2005, 15:R64-67.

38. Jones $E G$, Rubenstein JL: Expression of regulatory genes during differentiation of thalamic nuclei in mouse and monkey. J Comp Neurol 2004, 477:55-80.

39. Zhou CJ, Pinson Kl, Pleasure SJ: Severe defects in dorsal thalamic development in low-density lipoprotein receptor-related protein-6 mutants. J Neurosci 2004, 24:7632-7639.

40. Zeltser $L M$, Larsen CW, Lumsden A: A new developmental compartment in the forebrain regulated by Lunatic fringe. Nat Neurosci 2001, 4:683-684.

41. Gomez-Skarmeta J, de La Calle-Mustienes E, Modolell J: The Wnt-activated Xiro1 gene encodes a repressor that is essential for neural development and downregulates Bmp4. Development 2001, 128:551-560.

42. Matsumoto K, Nishihara S, Kamimura M, Shiraishi T, Otoguro T, Uehara M, Maeda Y, Ogura K, Lumsden A, Ogura T: The prepattern transcription factor Irx2, a target of the FGF8/MAP kinase cascade, is involved in cerebellum formation. Nat Neurosci 2004, 7:605-612.

43. Shimizu T, Hibi M: Formation and patterning of the forebrain and olfactory system by zinc-finger genes Fezf1 and Fezf2. Dev Growth Differ 2009, 51:221-231.

44. Giraldez AJ, Cohen SM: Wingless and Notch signaling provide cell survival cues and control cell proliferation during wing development. Development 2003, 130:6533-6543.
45. Johnston LA, Sanders AL: Wingless promotes cell survival but constrains growth during Drosophila wing development. Nat Cell Biol 2003, 5:827-833.

46. Chenn A, Walsh CA: Regulation of cerebral cortical size by control of cell cycle exit in neural precursors. Science 2002, 297:365-369.

47. Zechner D, Fujita Y, Hulsken J, Muller T, Walther I, Taketo MM, Crenshaw EB, Birchmeier W, Birchmeier C: beta-Catenin signals regulate cell growth and the balance between progenitor cell expansion and differentiation in the nervous system. Dev Biol 2003, 258:406-418.

48. Robu ME, Larson JD, Nasevicius A, Beiraghi S, Brenner C, Farber SA, Ekker SC: p53 activation by knockdown technologies. PLoS Genet 2007, 3 e78.

49. Gerety SS, Wilkinson DG: Morpholino artifacts provide pitfalls and reveal a novel role for pro-apoptotic genes in hindbrain boundary development. Dev Biol 2011, 350:279-289.

50. Kim NH, Kim HS, Kim NG, Lee I, Choi HS, Li XY, Kang SE, Cha SY, Ryu JK, Na JM, Park C, Kim K, Lee S, Gumbiner BM, Yook Jl, Weiss SJ: p53 and MicroRNA-34 are suppressors of canonical Wnt signaling. Sci Signal 2011, 4:ra71.

51. Brand M, Granato M, Nusslein-Volhard C: Keeping and raising zebrafish. In Zebrafish. Edited by: Nusslein-Volhard C, Dahm R. Oxford, UK: Oxford University Press; 2002:7-38.

52. Scholpp S, Delogu A, Gilthorpe J, Peukert D, Schindler S, Lumsden A: Her6 regulates the neurogenetic gradient and neuronal identity in the thalamus. Proc Natl Acad Sci USA 2009, 106:19895-19900.

53. Buckles GR, Thorpe CJ, Ramel MC, Lekven AC: Combinatorial Wnt control of zebrafish midbrain-hindbrain boundary formation. Mech Dev 2004, 121:437-447.

54. Itoh M, Kudoh T, Dedekian M, Kim CH, Chitnis AB: A role for iro1 and iro7 in the establishment of an anteroposterior compartment of the ectoderm adjacent to the midbrain-hindbrain boundary. Development 2002, 129:2317-2327.

55. Langheinrich U, Hennen E, Stott G, Vacun G: Zebrafish as a model organism for the identification and characterization of drugs and genes affecting p53 signaling. Curr Biol 2002, 12:2023-2028.

56. Scholpp S, Lohs C, Brand M: Engrailed and Fgf8 act synergistically to maintain the boundary between diencephalon and mesencephalon. Development 2003, 130:4881-4893.

57. Weidinger G, Thorpe CJ, Wuennenberg-Stapleton K, Ngai J, Moon RT: The Sp1-related transcription factors sp5 and sp5-like act downstream of Wnt/beta-catenin signaling in mesoderm and neuroectoderm patterning. Curr Biol 2005, 15:489-500.

58. Scholpp S, Brand M: Integrity of the midbrain region is required to maintain the diencephalic-mesencephalic boundary in zebrafish no isthmus/pax2.1 mutants. Dev Dyn 2003, 228:313-322.

59. Krauss S, Korzh V, Fjose A, Johansen T: Expression of four zebrafish wntrelated genes during embryogenesis. Development 1992, 116:249-259.

60. Krauss S, Concordet JP, Ingham PW: A functionally conserved homolog of the Drosophila segment polarity gene hh is expressed in tissues with polarizing activity in zebrafish embryos. Cell 1993, 75:1431-1444.

61. Ekker SC, Ungar AR, Greenstein P, von Kessler DP, Porter JA, Moon RT, Beachy PA: Patterning activities of vertebrate hedgehog proteins in the developing eye and brain. Curr Biol 1995, 5:944-955.

62. Concordet JP, Lewis KE, Moore JW, Goodrich LV, Johnson RL, Scott MP, Ingham PW: Spatial regulation of a zebrafish patched homologue reflects the roles of sonic hedgehog and protein kinase $A$ in neural tube and somite patterning. Development 1996, 122:2835-2846.

63. Carl M, Bianco IH, Bajoghli B, Aghaallaei N, Czerny T, Wilson SW: Wnt/ Axin $1 /$ betacatenin signaling regulates asymmetric nodal activation, elaboration, and concordance of CNS asymmetries. Neuron 2007, 55:393-405.

64. Toyama R, Curtiss PE, Otani H, Kimura M, Dawid IB, Taira M: The LIM class homeobox gene lim5: implied role in CNS patterning in Xenopus and zebrafish. Dev Biol 1995, 170:583-593.

65. Mercier P, Simeone A, Cotelli F, Boncinelli E: Expression pattern of two otx genes suggests a role in specifying anterior body structures in zebrafish. Int J Dev Biol 1995, 39:559-573.

66. Blader P, Fischer N, Gradwohl G, Guillemot F, Strahle U: The activity of neurogenin 1 is controlled by local cues in the zebrafish embryo. Development 1997, 124:4557-4569. 
67. Hashimoto H, Yabe T, Hirata T, Shimizu T, Bae Y, Yamanaka Y, Hirano T, Hibi M: Expression of the zinc finger gene fez-like in zebrafish forebrain. Mech Dev 2000, 97:191-195.

doi:10.1186/1749-8104-7-12

Cite this article as: Mattes et al: Wnt3 and Wnt3a are required for induction of the mid-diencephalic organizer in the caudal forebrain. Neural Development 2012 7:12.

Submit your next manuscript to BioMed Central and take full advantage of:

- Convenient online submission

- Thorough peer review

- No space constraints or color figure charges

- Immediate publication on acceptance

- Inclusion in PubMed, CAS, Scopus and Google Scholar

- Research which is freely available for redistribution

Submit your manuscript at www.biomedcentral.com/submit 